\title{
Energy loss and longitudinal wakefield of relativistic short proton bunches in electron clouds
}

\author{
O. Boine-Frankenheim, ${ }^{1,2}$ E. Gjonaj, ${ }^{2}$ F. Petrov, ${ }^{2}$ F. Yaman, ${ }^{2}$ T. Weiland, ${ }^{2}$ and G. Rumolo ${ }^{3}$ \\ ${ }^{1}$ GSI Helmholtzzentrum für Schwerionenforschung GmbH, Planckstraße 1, 64291 Darmstadt, Germany \\ ${ }^{2}$ Technische Universität Darmstadt, Schlossgartenstraße 8, 64289 Darmstadt, Germany \\ ${ }^{3}$ CERN, Geneva, Switzerland
}

(Received 21 December 2011; published 16 May 2012)

\begin{abstract}
The aim of our study is the numerical computation of the wakefield and energy loss per unit length for relativistic, short $(<10 \mathrm{~ns})$ proton bunches interacting with an electron cloud inside the beam pipe. We present analytical expressions for the energy loss in the impulse kick approximation. For the simulation of the wakefields a 2D self-consistent, electrostatic particle-in-cell (PIC) code is employed. Results for the energy loss and for the wakefields are presented for the parameter scope of the CERN LHC and SPS. For selected parameters the results are compared to a three-dimensional (3D) electromagnetic PIC code.
\end{abstract}

DOI: 10.1103/PhysRevSTAB.15.054402

PACS numbers: 29.27.Bd, 29.20.dk, 52.35.Qz

\section{INTRODUCTION}

Electron clouds limit the intensity of hadron beams in modern high energy synchrotrons and colliders (see e.g. [1]). In the CERN LHC effects related to electron clouds have been observed at different bunch spacings [2]. The operation with $25 \mathrm{~ns}$ bunch trains is presently limited by electron cloud effects. For electron clouds the main observations are usually a pressure rise in the warm regions and an increase of the beam screen temperature in the cold sections due to the additional heat load. Besides there are the beam-based observations, like, e.g., head-tail instabilities above a threshold bunch intensity or below a certain threshold bunch spacing. Electron cloud induced beam instabilities are also of concern for the design of the SIS-100 synchrotron, which is part of the FAIR project at GSI [3]. From the instability growth rate, one can estimate the cloud density, provided that one has a sufficiently accurate numerical model of the interaction of the single bunch with the cloud. This interaction results in a wakefield induced by the bunch in the electron cloud. There are also important observations below the instability threshold. In the SPS and in the LHC, one attributes the observed slow transverse emittance growth as well as the rf phase shift of individual bunches in the train to the effect of the electron cloud [4]. Both effects depend again on the wakefield induced by a short, relativistic bunch in the cloud. The present study has been motivated by observations in the CERN SPS and LHC where an increase in the shift of the synchronous phase $\Delta \phi_{s}$ with decreasing bunch spacing has been observed [2,5]. In the LHC rf phase shifts of the order $0.01^{\circ} \lesssim \Delta \phi \lesssim 1^{\circ}$ were

Published by the American Physical Society under the terms of the Creative Commons Attribution 3.0 License. Further distribution of this work must maintain attribution to the author(s) and the published article's title, journal citation, and DOI. observed. The observed shifts increase linearly with the bunch intensity. After extensive scrubbing runs with $50 \mathrm{~ns}$ bunch spacing the slope $\Delta \phi_{s} / N_{i}$ of the rf phase shift with the number of protons in the bunch $N_{i}$ gradually decreased over the period of the scrubbing run. After the scrubbing runs, the slope decreased by 1 order of magnitude [6]. In order to use the measured rf phase shift for a quantitative estimate of the electron cloud density analytical models for the energy loss of bunches in electron clouds are required. In this study we will present simple expressions for the energy loss and compare them to simulation results. We will concentrate on the simulation of the wakefield and on the resulting energy loss of an individual bunch in a train. Wakefields induced by a short, relativistic bunch in a preexisting, homogeneous electron cloud have already been obtained numerically in Ref. [7]. Numerical results for the "pinching" of the electron density caused by a LHC bunch were analyzed in Ref. [4]. Analytic expressions for wakefields and impedances in the framework of the dielectric response theory were obtained in Ref. [8]. The dielectric approach results in a vanishing longitudinal wakefield and energy loss for relativistic bunches. Therefore we will use a numerical model for the wakefield together with analytical expressions for the stopping power in the impulse kick approximation. In Sec. II we briefly describe the relation between the energy loss, the rf phase shift, and the longitudinal wakefield. The simplified model for the electron cloud's equation of motion is presented in Sec. III. An analytic expression for the energy loss of a short bunch within the impulse kick approximation is obtained in Sec. IV. In Sec. the modification of this expression due to electron space charge effects is discussed. The two numerical models used in the study are briefly described in Sec. V. The numerical results for the wakefields and the stopping power are presented in Sec. VI. 


\section{ENERGY LOSS AND RF PHASE SHIFT}

During beam storage, the rf phase shift $\Delta \phi$ in a rf bucket is

$$
\sin \left(\Delta \phi_{s}\right)=\frac{\Delta W_{p}}{q V_{\mathrm{rf}}}
$$

where $q$ is the ion charge, $V_{\mathrm{rf}}$ is the rf amplitude, and $\Delta W_{p}$ is the energy loss per particle and per turn. The measurement of $\Delta \phi_{s}$ can be used to gain information on the longitudinal impedance spectrum [9]. In LHC the observed dependence of the rf phase shift on the bunch spacing indicates that electron clouds can be the source of the energy loss. In general, the stopping power $S$ (total energy loss of the bunch per length unit) can be written as

$$
\frac{d W}{d s}=-\int \rho_{i}(\vec{r}) E_{z}(\vec{r}) d^{3} r \approx-q \int \lambda(z) E_{z}(z) d z,
$$

where $\rho_{i}$ is the bunch charge density, $E_{z}(z)$ is the longitudinal electric field induced by the bunch, $\lambda(z)$ is the line density of the bunch, and $q=e$ is the particle charge. $z=s-s_{0}$ is the longitudinal coordinate relative to the synchronous particle at $s_{0}$. The energy loss per particle and per turn is

$$
\Delta W_{p}=\frac{L}{N_{i}}\left\langle\frac{d W}{d s}\right\rangle
$$

where $L$ is the ring circumference, $N_{i}$ the number of protons in the bunch, and $\langle\cdots\rangle$ represents the stopping power averaged over the ring circumference. If the electric field induced by the bunch can be described in terms of a longitudinal impedance $Z^{\|}$, the energy loss per ion and per turn can be obtained from Eq. (2) as (see [10])

$$
\frac{\Delta W_{p}}{L}=-\frac{q^{2}}{N_{i} R} \int_{-\infty}^{\infty}|\lambda(\omega)|^{2} \operatorname{Re} Z^{\|}(\omega) d \omega,
$$

where $Z^{\|}$is the longitudinal ring impedance. For short bunches, the energy loss is independent of the bunch length and depends on the area under $\operatorname{Re} Z^{\|}(\omega)$ only. The present study focuses on the wakefield induced by the bunch in an electron cloud and the resulting stopping power. The question whether or not the obtained longitudinal wakefield can be described in terms of a longitudinal impedance is also of general relevance. However, we will not provide the answer to this question in the present study. The impedance, representing the accelerator environment and the cloud, should only depend on the initial electron cloud parameters, independent on the bunch profile and intensity. The stopping power will then depend on the total bunch charge $Q_{i}=$ $q N_{i}$ as $S \propto Q_{i}^{2}$. Thus, the scaling of the stopping power with the bunch intensity and length can already give an indication if an impedance description is possible for a certain bunch parameter regime. It is important to notice that the energy transferred by the bunch to the electrons is finally deposited in the pipe wall. The heat load (in $\mathrm{W} / \mathrm{m}$ ) is directly related to the stopping power via $c S / l_{b b}$, where $l_{b b}$ is the bunch distance.
In the following we will estimate the energy loss due to electron clouds from a simplified analytical model. The results from the analytic expression will be compared to the energy loss obtained from a simulation model. First, we will describe the model for the interaction of the bunch with the cloud.

\section{ELECTRON EQUATION OF MOTION}

The rigid bunch of velocity $v_{0} \approx c$ interacts with the electrons via its transverse electric field $E_{r}^{i}(r, z)$. Here we will ignore the beam's magnetic field as well as any magnetic field induced by the electrons. For a transverse Gaussian beam profile, we obtain for the electric field

$$
E_{r}^{i}(r, z)=\frac{q \lambda(z, t)}{2 \pi \epsilon_{0} r}\left[1-\exp \left(-\frac{r^{2}}{2 \sigma_{\perp}^{2}}\right)\right] .
$$

For the sake of simplicity, we assume a round beam of radius $a=2 \sigma_{\perp} . a$ is the radius of the rms equivalent constant beam profile. The line density of the bunch is assumed to be Gaussian with

$$
\lambda(z)=\frac{N_{i}}{\sqrt{2 \pi} \sigma_{z}} \exp \left(-\frac{z^{2}}{2 \sigma_{z}^{2}}\right),
$$

where $z=z_{0}-c t, N_{i}$ is the number of ions in the bunch, $\sigma_{z}$ is the rms bunch length. The resulting electron equation of motion is

$$
r^{\prime \prime}+\kappa^{2}(r, z) r=\frac{e E_{r}^{e}(r, z)}{m_{e} c^{2}},
$$

where $\kappa(r, z)$ represents the focusing force due to the beam's transverse electric field and $E_{r}^{e}$ is the electric field of the electron cloud. For $r<a$ and a constant beam profile, the focusing gradient is

$$
\kappa(z)=\frac{\sqrt{2 \lambda(z) r_{e}}}{a} .
$$

The electron space charge field $E_{r}^{e}(r, z)$ induced by the bunch in the cloud has to be obtained from Gauss law with the electron charge density $\rho_{e}(r, z)$.

\section{ENERGY LOSS OF SHORT BUNCHES}

First, we will ignore the effect of electron space charge. Furthermore, we will assume that the bunch length $\sigma_{z}$ is short relative to the electron oscillation length in the bunch center $\kappa^{-1}(0)=\kappa_{0}^{-1}$,

$$
\kappa_{0} \sigma_{z} \lesssim 1,
$$

and that the electrons are homogeneously distributed inside the beam pipe of radius $R_{p} \gg a$. In this case, the majority of the electrons will simply receive a transverse impulse kick from the passing bunch (see also Ref. [11]): 
$\Delta p_{\perp}(b)=\frac{1}{c} \int_{-\infty}^{\infty} F_{\perp}(b, s) d s, \quad F_{\perp}=-e E_{\perp}^{i}(b, s)$.

$b$ is the impact parameter or transverse distance between the electron and the beam axis. The total energy gain of the electrons per unit length is

$$
\frac{d W_{e}}{d s}=\frac{n_{e}}{2 m_{e}} \int_{0}^{R_{p}} 2 \pi \Delta p_{\perp}^{2}(b) b d b
$$

and the stopping power

$$
S=\frac{d W_{e}}{d s} \approx \frac{Q_{i}^{2} n_{e} r_{e}}{\varepsilon_{0}} \ln \left(\frac{R_{p}}{a}\right) .
$$

The corresponding rf phase shift per unit length is (assuming $\left.\Delta \phi_{s} \ll 1\right)$

$$
\frac{d \Delta \phi_{s}}{d s} \approx \frac{Q_{i} n_{e} r_{e}}{\varepsilon_{0} V_{r f}} \ln \left(\frac{R_{p}}{a}\right) .
$$

So far we have neglected the effect of dipole or quadrupole magnetic fields on the electrons. In a dipole field, the number of cyclotron oscillation per bunch length can be estimated from

$$
N_{c} \approx \frac{\omega_{c} \sigma_{z}}{2 \pi c},
$$

where $\omega_{c}$ is the cyclotron frequency $e B_{y} / m_{e}$ (see also Ref. [11]). For $N_{c} \gg 1$ we can assume that the electrons only respond along the magnetic field lines and the stopping power given by Eq. (12) is reduced by the factor

$$
\frac{1}{2 \pi} \int_{0}^{2 \pi} \sin ^{2} \theta d \theta=\frac{1}{2}
$$

\section{Electron cloud space charge}

In the following we will present a simple model for the effect of electron space charge on the stopping power. This model is based on Mulser's oscillator model [12]. The force exerted on the electrons by a relativistic bunch is purely transverse. Therefore the stopping power and the longitudinal wakefield obtained from the linear, dielectric plasma theory would both be zero (see, e.g., Refs. $[13,14]$ ). This can also be seen from the longitudinal electron cloud impedance obtained analytically from the dielectric theory in Ref. [8]. Both, the space charge impedance seen by the beam as well as the contribution of the electrons, go to zero in the relativistic limit. This is because of the vanishing longitudinal bunch force component. In the relativistic limit the longitudinal wakefield is created by the transverse bunch force causing the pinching of the electrons. Therefore, instead of the dielectric theory, we use Mulser's oscillator model [12] in which the energy loss of the bunch is obtained from the energy transferred into plasma waves. During the passage of the bunch through the cloud, the bunch excites plasma oscillators along its path $s=c t$ according to

$$
\delta^{\prime \prime}+\frac{\omega_{p e}^{2}}{c^{2}} \delta=\kappa^{2}(b, z) b,
$$

where $\delta$ is the oscillator offset and

$$
\omega_{p e}=\sqrt{\frac{e^{2} n_{e}}{m_{e} \varepsilon_{0}}}
$$

is the plasma frequency. $b$ is the impact parameter or transverse distance between the oscillator and the passing bunch. The position of the oscillator is assumed to remain constant during the interaction. The resulting oscillator amplitude at $s=\infty$ is determined by

$$
\hat{\delta}(b)=\frac{b}{\kappa_{e}} \int_{-\infty}^{\infty} \kappa(b, s)^{2} \cos \left(\kappa_{e} s\right) d s,
$$

where $\kappa_{e}=\omega_{p e} / c$ is the inverse "dynamical Debye length." The energy loss per unit length can be calculated from the energy transferred into plasma waves behind the bunch,

$$
\frac{d W_{e}}{d s}=\frac{1}{2} m_{e} n_{e} \omega_{p e}^{2} \int_{0}^{R_{p}} 2 \pi \hat{\delta}^{2} b d b,
$$

and the stopping power for $R_{p} \gg a$ results as

$$
S=\frac{d W_{e}}{d s} \approx \frac{Q_{i}^{2} \kappa_{e}^{2}}{4 \pi \varepsilon_{0}} \ln \left(\frac{R_{p}}{a}\right) \exp \left(-\kappa_{e}^{2} \sigma_{z}^{2}\right)
$$

which is exactly Eq. (12) multiplied by an exponential factor. For electron cloud densities exceeding $\kappa_{e} \sigma_{z} \approx 1$, the stopping power is reduced by the plasma shielding effect of the cloud.

\section{SIMULATION MODELS}

In order to go beyond the impulse kick approximation and to study the effect of the self-consistent electron space charge field, a two-dimensional (2D), electrostatic (ES) particle-in-cell (PIC) code was employed. In the 2D model the electrons evolve in a $(x, y)$ plane perpendicular to the bunch direction of motion. Poisson's equation is solved in 2D for the electrostatic potential $\phi(x, y)$ each time step using the electron charge density $\rho_{e}(x, y, t)$ and the known bunch density $\rho_{i}\left(x, y, z=z_{0}-c t\right) . \phi=0$ is set at the pipe boundary. At the beam pipe electrons are either elastically reflected, absorbed, or multiplied according to the secondary emission yield (SEY) given in Ref. [15].

The $2 \mathrm{D}$ electron density is stored every time step $t_{j}$. At the end of a simulation run, a $3 \mathrm{D}$ electron density is constructed from the stored 2D slices $\rho_{e}(x, y, z=$ $\left.z_{0}-c t_{j}\right)$. The $3 \mathrm{D}$ potential $\phi(x, y, z)$ is obtained numerically from the Poisson equation using $\rho_{e}(x, y, z)$. In the case of a circular beam pipe and without an external $B$ field, a Poisson solver in cylindrical $(r, z)$ coordinates is sufficient. From the derivative of the potential $\phi(x, y, z)$ we obtain the longitudinal electric field as 


$$
E_{z}(z)=-\left\langle\frac{\partial \phi(x, y, z)}{\partial z}\right\rangle,
$$

where $\langle\cdots\rangle$ indicates the average over the transverse beam profile. The stopping power is obtained from the longitudinal electric field and Eq. (2). For selected parameters, the stopping power and the electric field obtained from the 2D model were compared to simulation results obtained from the three-dimensional (3D) electromagnetic (EM) PIC code VORPAL [16]. In VORPAL a short, single bunch penetrates an initially homogeneous electron cloud in a cylindrical pipe. At the pipe wall the electrons are simply absorbed.

\section{SIMULATION RESULTS}

In our simulation we will first study the stopping power in an initially homogenous, low density $\left(\$ 10^{13} \mathrm{~m}^{-3}\right)$ electron cloud. The results are compared to Eq. (12). Afterwards we will analyze electron space charge effects for higher cloud densities. The results are compared to Eq. (20). The validity of the $2 \mathrm{D}$ electrostatic model is checked by comparing the longitudinal wakefields from the 2D and the 3D simulations. Finally, we compare the stopping power and wakefields obtained for a homogeneous cloud with the more realistic situation of a bunch interacting with a saturated electron cloud generated by the previous bunches.

In our studies we use two parameter sets. The first one is close to the LHC parameters at injection energy with the rms bunch length $\sigma_{z}=0.1 \mathrm{~m}$, beam radius $a=0.002 \mathrm{~m}$, and pipe radius $R_{p}=0.02 \mathrm{~m}$. The second set is close to the SPS parameters at extraction energy with the longer rms bunch length $\sigma_{z}=0.25 \mathrm{~m}$, beam radius $a=0.004 \mathrm{~m}$, and pipe radius $R_{p}=0.04 \mathrm{~m}$. In both cases the number of protons per bunch is chosen as $N_{i}=10^{11}$ and the velocity of the bunches is set to $c$.

\section{A. Low electron densities}

For the LHC parameter, set the stopping power obtained from Eq. (12) divided by the number of bunch particles $N_{i}$ is shown in Fig. 1 as a function of $N_{i}$. The electron density is chosen as $n_{e}=10^{12} \mathrm{~m}^{-3}$. The analytic result is compared to the stopping power obtained by solving Eq. (7) numerically (see Sec. V). For $N_{i}=10^{11}$ the electron density profile from the simulation is shown in Fig. 2. The result is very close to the plots shown in Ref. [4], where also the characteristic stripe structure seen in Fig. 2 is analyzed in detail. Electron space charge is included in the simulations, but its does not affect the stopping power and the wakefield for $n_{e}=10^{12} \mathrm{~m}^{-3}$. For the electron density shown in Fig. 2 the electric field is obtained from Poisson's equation. The resulting longitudinal electric field is shown in Fig. 3. Here we also compared the results from the $2 \mathrm{D}$ and the $3 \mathrm{D}$ simulations. Both simulation models lead to the same stopping power and wakefield.

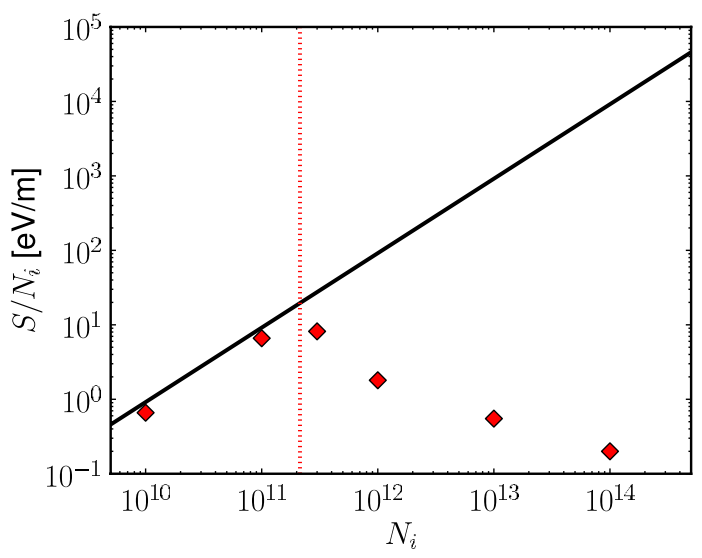

FIG. 1. Stopping power as a function of the number of particles in the bunch. The analytic result obtained from Eq. (12) is represented by the solid curve. The symbols represent the results obtained from the simulation. The red dashed line corresponds to $\kappa_{0} \sigma_{z}=10$.

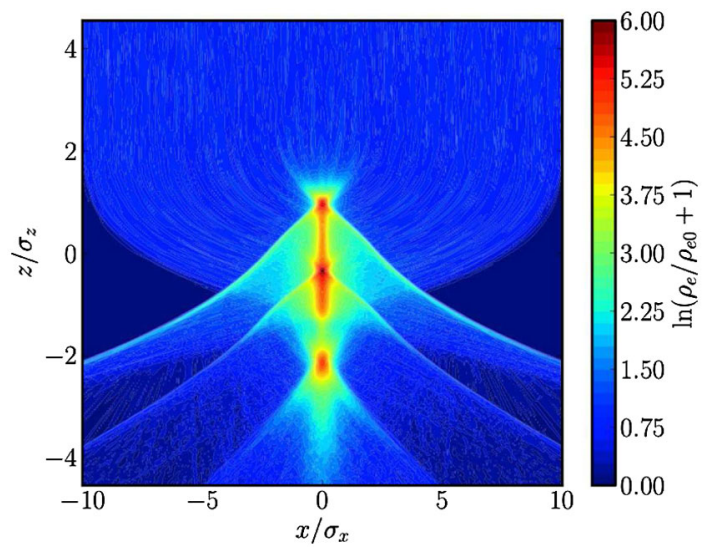

FIG. 2. Electron cloud density contour obtained from the tracking simulation for LHC parameters. The initial electron cloud charge density $\rho_{e 0}=e n_{e}$, with $n_{e}=10^{12} \mathrm{~m}^{-3}$, is used to normalize the color scale.

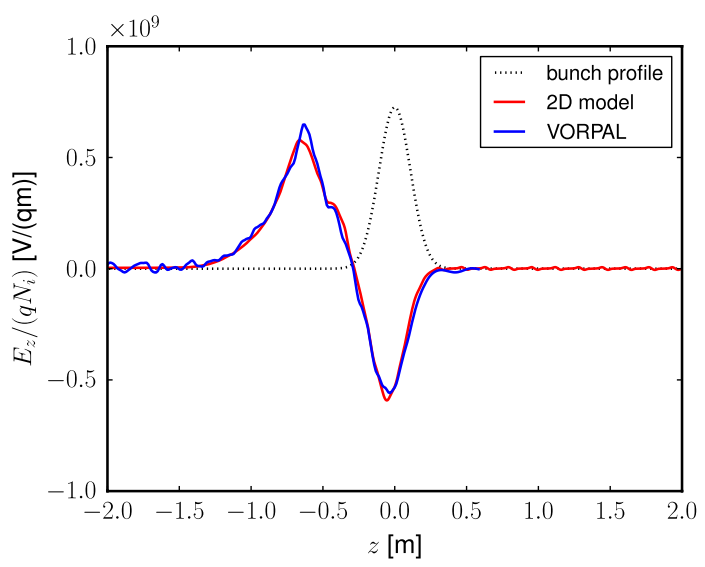

FIG. 3. Longitudinal electric field obtained from the 2D ES and the 3D EM simulations for $n_{e}=10^{12} \mathrm{~m}^{-3}$. 


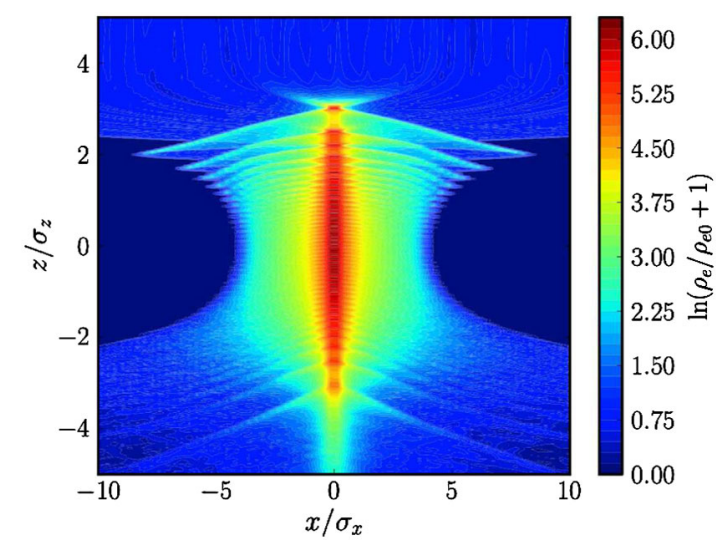

FIG. 4. Electron cloud density contour obtained from the tracking simulation for a larger number of particles per bunch $N_{i}=10^{13}$. The initial electron cloud charge density $\rho_{e 0}=e n_{e}$, with $n_{e}=10^{12} \mathrm{~m}^{-3}$, is used to normalize the color scale.

The comparison of the analytical and numerical results shown in Fig. 1 indicates that the momentum kick approximation is valid for $\kappa_{0} \sigma_{z} \lesssim 10$. For $\kappa_{0} \sigma_{z} \geq 10$ the energy loss per particle decreases (see Fig. 1) because of the dominant trapping of electrons in the beam potential. If particle trapping is important, the momentum kick approximation leading to Eq. (12) is not valid. Electrons that are trapped and detrapped adiabatically do not exchange net energy with the bunch.

As an example for this trapping regime, we show in Fig. 4 the electron density profile obtained from the simulation for $N_{i}=10^{13}$, which corresponds to $\kappa_{0} \sigma_{z} \approx 50$. Compared to Fig. 2 the density profile is more symmetric about the bunch center at $z=0$ which results in a more symmetric wakefield and consequently in a smaller energy loss (as also seen in Fig. 1).

For long ( $>10 \mathrm{~ns}$ ) and intense proton or heavy-ion bunches, like in the projected SIS-100 at GSI, the energy loss will be much lower than predicted by Eq. (12) and the rf phase shift might be too low to be measured.

For both the LHC and the SPS parameter sets, the kick approximation is valid and we obtain from Eq. (12) with $n_{e}=10^{12} \mathrm{~m}^{-3}$ the stopping power $S \approx 12 \mathrm{eV} / \mathrm{m}$ for the energy loss per proton and unit length, which corresponds to $\Delta \phi_{s} \approx 2 \times 10^{-4} \mathrm{deg} / \mathrm{m}$, for $V_{\mathrm{rf}}=3.5 \mathrm{MV}$. For the LHC this would correspond to $\Delta \phi_{s} \approx 0.5 \mathrm{deg}$, assuming the cloud would cover $10 \%$ of the ring circumference. The order of magnitude agrees with the LHC observations [6].

\section{B. High electron densities}

For the SPS parameter set, the stopping power obtained from Eq. (20) as a function of the electron cloud density is shown in Fig. 5. The symbols represent the simulation results and the vertical line indicates that the dynamical Debye length $\kappa_{e}^{-1}$ is equal to the rms bunch length $\sigma_{z}$. One can observe that the stopping power from the simulations starts to drop at $\kappa_{e} \sigma_{z} \approx 1$. However, Eq. (20) underestimates the

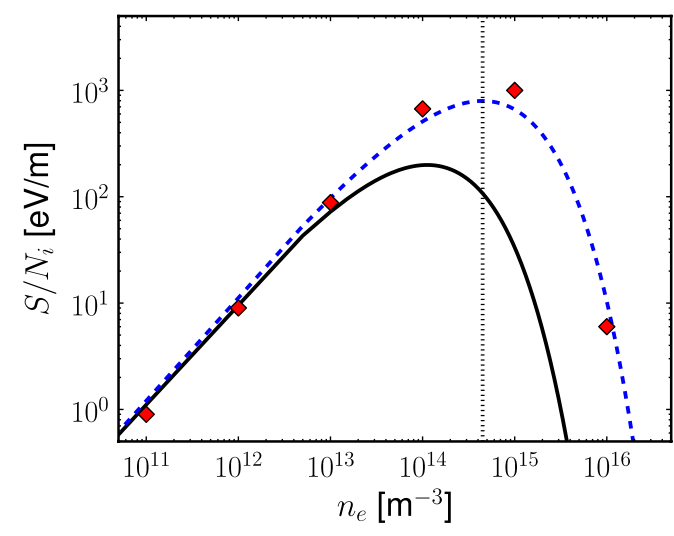

FIG. 5. Stopping power as a function of the electron density. The analytic results obtained from Eq. (20) for $\sigma_{z}=0.25 \mathrm{~m}$ is represented by the solid curve. The symbols represent the results obtained from the simulations. The vertical, dotted line corresponds to $\kappa_{e} \sigma_{z}=1$. The dashed blue curve corresponds to Eq. (20) for $\sigma_{z} \rightarrow \sigma_{z} / 2$.

simulation results for electron densities above this value. The simple, linear oscillator model behind Eq. (20) cannot correctly reproduce the coupling of the LHC bunch to the plasma modes in the beam pipe. Still, the analytical result [Eq. (20)] is useful to estimate the onset of electron space charge effects. It is worth noting that Eq. (20) with the substitution $\sigma_{z} \rightarrow \sigma_{z} / 2$ reproduces the simulation results very well, also in the case of the shorter LHC bunch. For electron cloud densities above $\kappa_{e} \sigma_{z} \approx 1$, the bunch excites undamped plasma waves in the electron cloud. For an initial $n_{e}=10^{16} \mathrm{~m}^{-3}\left(\kappa_{e} \sigma_{z} \approx 5\right)$, the contour plot of the electron cloud density obtained from the 2D ES simulation is shown in Fig. 6. One can clearly see the density waves launched behind the bunch. The weak density fluctuations in front of the bunch are caused by the intrinsic numerical noise of the PIC code for high densities. The resulting longitudinal electric field is shown in Fig. 7. The wavelength corresponds

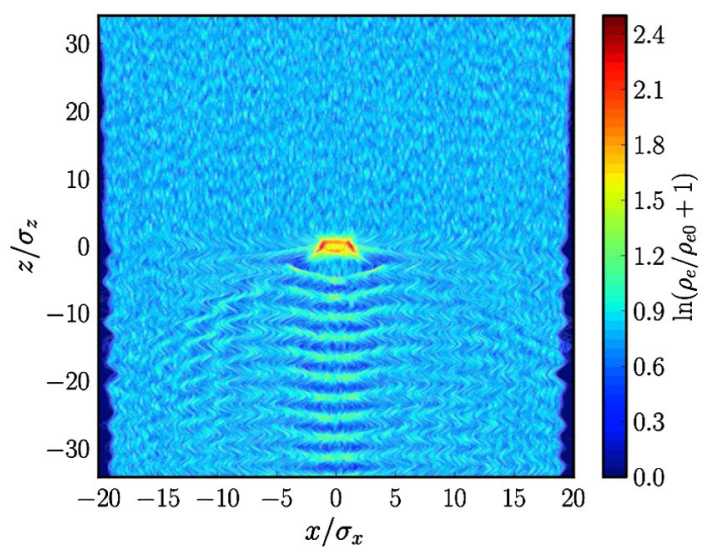

FIG. 6. Electron cloud density contour obtained from the 2D ES simulation for an electron cloud density $n_{e}=10^{16} \mathrm{~m}^{-3}$. The initial electron cloud charge density $\rho_{e 0}=e n_{e}$, with $n_{e}=$ $10^{16} \mathrm{~m}^{-3}$, is used to normalize the color scale. 


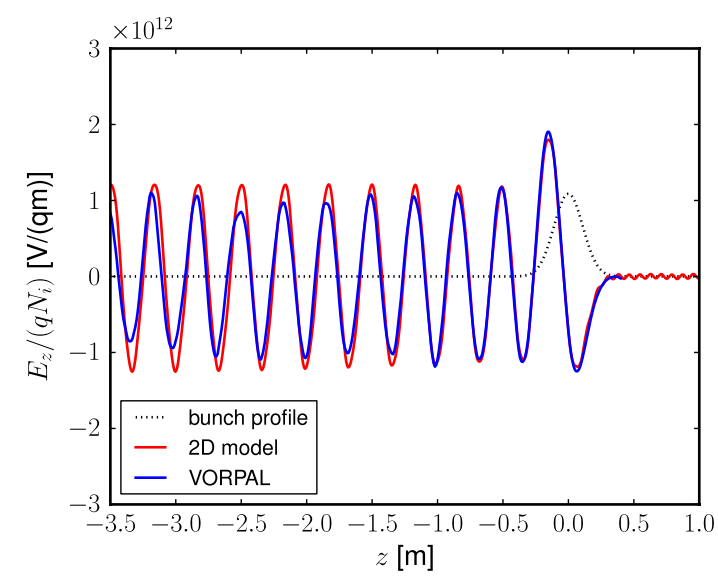

FIG. 7. Longitudinal electric field obtained from the 2D ES and the $3 \mathrm{D}$ EM simulations for $n_{e}=10^{16} \mathrm{~m}^{-3}$.

very well to the expected $2 \pi \kappa_{e} \approx 0.3 \mathrm{~m}$, which is the oscillation period of a plasma oscillation times the beam's velocity $c$. In the plot we compare the wakefields from the 2D and the 3D simulations for $n_{e}=10^{16} \mathrm{~m}^{-3}\left(\kappa_{e} \sigma_{z} \approx 5\right)$. Again both simulation models lead to the same stopping power and wakefield. Thus, we can conclude that for the target of the present study the relatively simple 2D ES model describes the interaction of a bunch with an electron sufficiently well.

It is important to note that according to electron buildup simulations (see also the next subsection) electron cloud densities of the order of $10^{15}-10^{16} \mathrm{~m}^{-3}$ will not be reached, because they are well above the typical saturation densities in a beam pipe. In order to load a stationary, dense cloud into the simulation code, we have to set the radial electric field at the pipe boundary to zero $E_{r}\left(R_{p}\right)=0$ or, equivalently, use a homogeneous ion background. Only in this case the dense electron cloud will be stationary (without the passing bunch). For the boundary condition $\phi\left(R_{p}\right)=0$ and without an ion background, cloud densities of $10^{15}-10^{16} \mathrm{~m}^{-3}$ would disintegrate before a wakefield could develop.

\section{Saturated cloud density}

So far we ignored the effect of the preceding bunches on the stopping power of an individual bunch. The saturated electron cloud density seen by a bunch at the end of a train is not homogeneous, as assumed so far. The maximum electron density is limited by the electron's space charge potential. The electron density grows until the space charge potential of the cloud exceeds the initial kinetic energy $E_{s}$ of the secondary electrons. The newly created electrons cannot penetrate into the electron cloud and remain near the pipe wall. The density of a homogeneous, saturated cloud can be easily obtained as (see also Ref. [1])

$$
n_{e s} \approx \frac{E_{s}}{\pi m_{e} c^{2} R_{p}^{2} r_{e}} .
$$

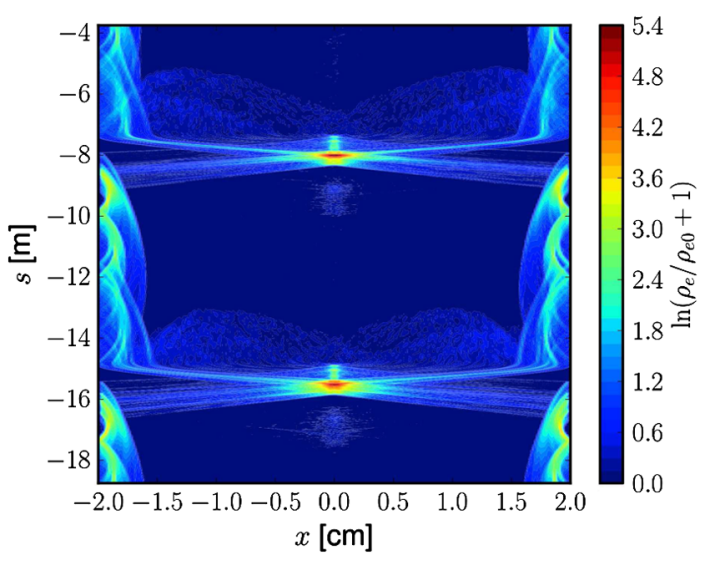

FIG. 8. Contour plot of the saturated electron cloud density obtained from the 2D simulation for LHC parameters. The electron cloud charge density $\rho_{e 0}=e n_{e}$, with $n_{e}=10^{13} \mathrm{~m}^{-3}$, is used to normalize the color scale.

For LHC parameters and $E_{s} \approx 2 \mathrm{eV}$ we obtain $n_{e s} \approx$ $10^{12} \mathrm{~m}^{-3}$. However, Eq. (22) only describes the initial stage of the saturation process, when the cloud is still homogeneous. If we assume that during the later stages an inhomogeneous cloud density with a dense layer of thickness $d \ll R_{p}$ at the wall develops, then the average cloud density $n_{e s}$ can be higher than Eq. (22). The realistic saturated cloud density must be obtained from numerical simulations. Within our 2D simulation model we performed simulation studies for the example case of a LHC bunch train with $25 \mathrm{~ns}$ bunch spacing. The electron reflection and generation at the wall is treated using the SEY given in Ref. [15]. For the chosen example parameters, we obtain an averaged electron density of $n_{e s} \approx 10^{13} \mathrm{~m}^{-3}$. The electron density for the saturated cloud is shown in Fig. 8 as a function of the coordinates $(x, s)$. It can be seen that the freshly generated electrons remain close to the wall before they are accelerated in the bunch potential. Because of their large impact factor $b=R_{p}$, the electrons cross the axis well behind the bunch head. The large density spots around the axis $(x=0)$ correspond to this crossing point. The resulting longitudinal electric field for one bunch is shown in Fig. 9. Compared to the electric field created in a homogeneous cloud, the electric field minimum is shifted backwards. This can be explained by the dominant contribution by electrons initially located near the wall. The obtained stopping power is smaller by roughly a factor 3 compared to the corresponding homogeneous cloud density (taken at the head of the bunch). The reduction in the stopping power can be explained by the concentration of the electrons at the wall. If $n_{e}$ is the average electron density in the pipe, the local electron density near the pipe is

$$
n_{e d}=n_{e} \frac{R_{p}^{2}}{R_{p}^{2}-\left(R_{p}-d\right)^{2}} \approx n_{e} \frac{R_{p}}{2 d} .
$$




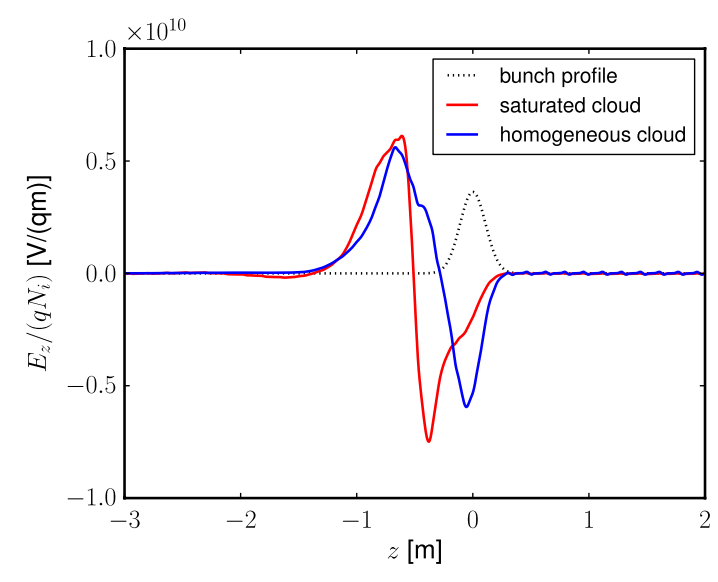

FIG. 9. The longitudinal electric field obtained from the 2D simulations induced by a LHC bunch in the saturated cloud and in the corresponding homogeneous cloud $\left(n_{e} \approx 10^{13} \mathrm{~m}^{-3}\right)$. Shown is the electric field induced by the lower bunch in Fig. 8.

If we make the substitution $a \rightarrow R_{p}-d$ with $d \ll R_{p}$ in Eq. (12), we obtain for the stopping power in a saturated electron cloud

$$
S_{s} \approx \frac{Q_{i}^{2} \kappa_{e}^{2}}{8 \pi \varepsilon_{0}}
$$

$S_{s}$ is smaller by the factor $2 \ln \left(R_{p} / a\right)$ than the stopping power in a homogeneous cloud of the same average density [Eq. (12)]. For SPS and LHC parameters, this corresponds to a maximum reduction of the stopping power by a factor 4.6. A finite layer thickness $d$ will result in a smaller reduction. For example, a layer thickness of $d=0.3 R_{p}$ would result in a reduction factor of only 3.2, which is closer to the stopping power obtained from the simulations. It is important to note that the effect of electron space charge on the stopping power cannot be analyzed separately in the simulations, because it also determines the saturation density.

\section{CONCLUSIONS}

The energy loss and wakefield of a short, relativistic proton bunch in an electron cloud has been studied within a 2D electrostatic simulation model. The results were compared to 3D full EM simulations. For SPS and LHC-type bunches, the 2D ES model is found to be sufficient to predict the stopping power and the longitudinal wakefield induced in an electron cloud. We found that for sufficiently short bunches or $\kappa_{0} \sigma_{z} \lesssim 10$ the energy loss in a homogeneous cloud can be described very well by an analytic formula. For $\kappa_{0} \sigma_{z} \lesssim 10$ we find that the stopping power scales according to $\propto Q_{i}^{2}$, which would be equivalent to the effect of a longitudinal resistive impedance. For an example electron cloud density of $10^{12} \mathrm{~m}^{-3}$ and assuming that the cloud would cover $10 \%$ of the ring circumference, our analytic expression predicts an rf phase shift of
$\Delta \phi_{s} \approx 0.5 \mathrm{deg}$ in LHC. The order of magnitude agrees with the LHC observations. Detailed comparisons of the presented theoretical models with the SPS and LHC observations will be the subject of future studies. For electron densities well above the typical $10^{11}-10^{13} \mathrm{~m}^{-3}$ or $\kappa_{e} \sigma_{z} \geq 1$, the "shielding" effect of the space charge field of the electrons reduces the stopping power. This reduction can be described by a simple exponential multiplication factor. A detailed study of the structure of the wakefield together with analytic expressions remains as the subject for future work. An analytic description of the stopping power of a short bunch in a saturated cloud is more difficult. In a saturated cloud the electrons are concentrated at the pipe wall. From a simple analytic expression we obtain the stopping power in a saturated cloud, which is lower compared to the value in the equivalent homogeneous cloud. The reduction in the stopping power is confirmed by $2 \mathrm{D}$ simulation results for bunch trains interacting with a saturated cloud.

\section{ACKNOWLEDGMENTS}

This work was supported by the BMBF under Contract No. 06DA9022I (Fedor Petrov and Fatih Yaman).

[1] F. Zimmermann, Phys. Rev. ST Accel. Beams 7, 124801 (2004).

[2] G. Rumolo, G. Arduini, V. Baglin, H. Bartosik, N. Biancacci, P. Baudrenghien, G. Bregliozzi, P. Chiggiato, S. Claudet, R. D. Maria, M. Favier, C. Hansen, J. M. Jimenez, V. Kain, G. Lanza, K. Li, H. M. Cuna, G. Papotti, T. Pieloni, F. Roncarolo, B. Salvant, E. Shaposhnikova, R. Steinhagen, L. Tavian, D. Valuch, W. Venturini, F. Zimmermann, U. Iriso, O. Dominguez, E. Koukovini-Platia, N. Mounet, C. Zannini, and C. Bhat, in IPAC2011 (2011), p. 2862.

[3] F. Petrov, O. Boine-Frankenheim, and T. Weiland, in IPAC2011 (2011), p. 724.

[4] G. Franchetti, I. Hofmann, and F. Zimmermann, Phys. Rev. ST Accel. Beams 12, 124401 (2009).

[5] E. Shaposhnikova, in CERN-GSI Electron Clouds Workshop (CERN, Geneva, 2011).

[6] J. Esteban-Müller and E. Shaposhnikova (private communication).

[7] G. Rumolo and F. Zimmermann, Phys. Rev. ST Accel. Beams 5, 121002 (2002).

[8] A. Al-Khateeb, R. W. Hasse, O. Boine-Frankenheim, and I. Hofmann, New J. Phys. 10, 083008 (2008).

[9] E. Shaposhnikova, T. Bohl, A. Hofmann, and T. Linnecar, in Proceedings of the 9th European Particle Accelerator Conference, Lucerne, 2004 (EPS-AG, Lucerne, 2004), pp. 1909-1911.

[10] K.Y. Ng, Physics of Intensity Dependent Beam Instabilities (World Scientific, Singapore, 2006).

[11] G. Rumolo, F. Ruggiero, and F. Zimmermann, Phys. Rev. ST Accel. Beams 4, 012801 (2001). 
[12] P. Mulser, K. Niu, and R. Arnold, Nucl. Instrum. Methods Phys. Res., Sect. A 278, 89 (1989).

[13] T. Peter and J. Meyer-ter Vehn, Phys. Rev. A 43, 1998 (1991).

[14] O. Boine-Frankenheim, Phys. Plasmas 3, 1585 (1996).
[15] R. Cimino, I. R. Collins, M. A. Furman, M. Pivi, F. Ruggiero, G. Rumolo, and F. Zimmermann, Phys. Rev. Lett. 93, 14801 (2004).

[16] C. Nieter and J.R. Cary, J. Comput. Phys. 196, 448 (2004). 